\title{
Actividad diaria de forrajeo en Polybia diguetana Buysson 1905 (Hymenoptera, Vespidae, Polistinae) Daily foraging activity of Polybia diguetana Buysson 1905 (Hymenoptera, Vespidae, Polistinae)
}

\author{
Alejandra González-Moreno ${ }^{10}$, Jorge L. Leirana-Alcocer ${ }^{* 2}$ 이 y José C. Cervera-Herrera ¿º \\ 1. División de Estudios de Posgrado e Investigación, Instituto Tecnológico de Conkal, Yucatán, México \\ 2 Facultad de Medicina Veterinaria y Zootecnia. Universidad Autónoma de Yucatán, Yucatán, México \\ *Autor de correspondencia: jleirana@correo.uady.mx \\ Recibido: 09 julio de 2019 \\ Aceptado: 25 de septiembre de 2019 \\ Publicación en línea: 31 de diciembre 2019
}

\section{Palabras clave: \\ avispas papeleras; actividad diurna; enemigos naturales; Polistinae}

Key words:

paper wasps; diel activity; natural enemies; Polistinae

\section{Resumen}

Es importante estudiar la actividad de forrajeo de Polistinae para entender mejor su impacto en los ecosistemas. En este trabajo se describe la actividad de un nido en fase de post-emergencia de Polybia diguetana en relación a la temperatura y humedad relativa. El área de estudio fue un campus universitario en Mérida, México, con clima cálido subhúmedo. Se muestreó en las siguientes fechas de 2019: enero 30 y 31, febrero 5 y 6, y abril 9 y 10, el 9 llovió de 9:00 a 11:45 horas. Se tomó un video del nido de 3:40 min de duración cada media hora, iniciando a las 7:30 y finalizando a las 14:45 horas. En total fueron filmados 83 videos con una duración acumulada de 308,5 minutos. Se midió la temperatura $\left({ }^{\circ} \mathrm{C}\right)$ y humedad relativa a tres metros del nido al iniciar cada video. Se realizaron análisis de correlación parcial con temperatura y humedad como variables independientes y frecuencias de despegues y aterrizajes como dependientes, el 9 de abril se excluyó del análisis por el posible efecto de la lluvia. En el día lluvioso los aterrizajes y despegues fueron más frecuentes a las 7:30 que hubo la temperatura más alta y la humedad relativa más baja; en los otros días los despegues fueron más frecuentes a las 11:00 y los aterrizajes a las 14:00. La temperatura se correlacionó positivamente tanto con los despegues $(r=0,21$, $p=0,05)$ como con los aterrizajes $(r=0,44, p<0,0001)$. La humedad relativa se correlacionó negativamente con los aterrizajes $(r=-0,27, p=0,01)$. La temperatura fue el factor que más influyó las actividades de forrajeo, la humedad relativa lo hizo en menor medida. La presencia de lluvias modificó el patrón, tanto por el aumento en la humedad relativa como por el riesgo de impacto sobre las forrajeras.

\section{Abstract}

It is important to study the foraging activity of Polistinae in order to understand their impact on ecosystems. In this work we describe the activity of one nest in post-emergence state of Polybia diguetana in relation to temperature and relative humidity. The study area is a university campus in Mérida, México, with warm sub - humid climate. Sampling were taken in the following dates of 2019: January 30 and 31, February 5 and 6 and April 9 and 10, the 9 it rained from 9:00 to 11:45 hours. A video lasting 3:40 min was taken every half an hour, beginning at 7:30 and finishing at 14:45. Eighty-three videos were recorded with an accumulated time of 308.5 minutes. When the video recording was beginning, temperature $\left({ }^{\circ} \mathrm{C}\right)$ and relative humidity (\%) were measured at a distance of 3 meters from the nest. Partial correlation analysis were carried out, with temperature and relative humidity as independent variables and wasp departures and arrivals frequencies as response variables, April 9th was excluded from this analysis for excluding the effect of the rain. In the rainy days the highest frequency of arrivals and departures was at 7:30, when the temperature was the highest and the humidity the lowest. In other days departure frequency was higher at 11:00 and arrivals at 14:00. Temperature was positively correlated to departures $(r=0.21, p=0.05)$ and arrivals $(r=0.44, p<0.0001)$. Relative humidity was $c$ orrelated to arrivals only $(r=-0.27, p=0.01)$. Temperature was the main factor influencing foraging activity, and humidity did in lesser degree. The pattern was different in the rainy day, due to raises in humidity and the risk of being impacted by raindrops. 
Las avispas sociales del género Polybia (Vespidae, Polibinae) prestan importantes servicios ambientales al depredar fitófagos que afectan tanto a plantas silvestres como cultivos (Prezoto et al., 2019), de igual manera colaboran en la polinización de algunas plantas (Afik et al., 2014). En su papel de depredadoras pueden limitar las poblaciones de sus presas y moldear su evolución (Hunt, 2007; Hernández et al., 2009).

Para subsistir las avispas de la subfamilia Polistinae requieren de agua para la construcción y refrigeración del nido, proteína animal para alimentar a las larvas, fibras de origen vegetal para construir su nido y líquidos azucarados para alimentar a los adultos (Canevazzi y Noll, 2011); la demanda de estos recursos determinan en gran parte el ritmo de actividad de las obreras, pero también pueden verse influidas por factores ambientales como temperatura, humedad relativa y la precipitación (Kasper et al., 2008; Detoni et al., 2015).

La temperatura es el factor ambiental que más influye en las actividades de éstos y de otros insectos, a bajas temperaturas ambientales el metabolismo es demasiado lento y los individuos no pueden activar sus músculos torácicos (Stabentheiner et al., 2012), mientras que a temperaturas muy altas se requiere forrajear mayores cantidades de agua que es utilizada para refrigerar el nido por evaporación (Hernández et al., 2009). La lluvia es otro factor que puede limitar la actividad de las avispas sociales, se ha documentado que las obreras del género Vespula disminuyen sus actividades hasta en un $30 \%$ durante las horas de lluvia (Kasper et al., 2008). En este trabajo se describieron las actividades de forrajeo de Polybia diguetana durante cinco días sin lluvias y su relación con la temperatura y la humedad relativa, y el patrón de actividades en un día con tres horas de lluvia.

El área de estudio fue el Campus de Ciencias Biológicas y Agropecuarias de la Universidad Autónoma de Yucatán, que se encuentra a $15,5 \mathrm{~km}$ al sur de la ciudad de Mérida, México. El clima es cálido subhúmedo con precipitaciones principalmente en los meses de verano (junio a octubre), la precipitación y la temperatura anuales promedio son de $1282 \mathrm{~mm}$ y de $26,3^{\circ} \mathrm{C}$ respectivamente (Comisión Nacional del Agua, 2010).

La vegetación está compuesta principalmente por jardines, en las que se combinan árboles exóticos como Delonix regia con otros nativos como Tabebuia rosea, Tecoma stans y Ceiba pentandra, también existen algunos fragmentos ocupados por relictos de la vegetación original a los que no se le presta ningún 201 cuidado, los árboles que dominan aquí son típicos de julio- diciembre de 2019 las selvas yucatecas: Bursera simaruba, Gymnopodium floribundum, Leucaena leucocephala y Caesalpinea sp., entre otros.

El objeto de este estudio fue un nido de Polybia diguetana en fase de post-emergencia de acuerdo a Jeanne (1972), de forma semiesférica, de aproximadamente $20 \mathrm{~cm}$ de diámetro; y que se encontraba a una altura de $10 \mathrm{~m}$ del suelo adherido a la cornisa del edificio del departamento de ecología tropical.

Los días del estudio fueron el 30 y 31 de enero, 5 y 6 de febrero y 9 y 10 de abril de 2019; el día 9 de abril llovió de 9 a 11:45 a.m. Cada día se filmó el nido con una cámara Canon EOS T6 con un zoom de 55250 mm, situada aproximadamente a tres metros del nido.

Se tomaron videos cada 30 minutos iniciando aproximadamente a las 7:30 am y finalizando a las 14:45 horas; cada video tuvo una duración de 3:40 minutos. En total se realizaron 83 videos para un acumulado de 308,5 minutos de filmación. Al momento de iniciar cada video se midieron la temperatura en ${ }^{\circ} \mathrm{C} y$ humedad relativa del aire (\%) utilizando un datalogger Elitech RC $51 \mathrm{H}$, colocado a menos de tres metros de la ubicación del nido.

Se analizaron los videos y se contabilizaron la cantidad de obreras que abandonaban ("despegues" de aquí en adelante) y las que regresaban al nido ("aterrizajes" de aquí en adelante) en cada video.

Se realizaron análisis de correlación parcial en los que se incluyó a la temperatura y humedad relativa como variables independientes y a los despegues y aterrizajes como variables dependientes; el paquete estadístico que se usó fue Statgraphics Centurion XV (StatPoint, Inc., 2007). Para este análisis se excluyeron los datos del 9 de abril, para evitar confundir el efecto de la lluvia en el comportamiento de los véspidos (Kasper et al., 2008). Al terminar el período de muestreo, tres individuos fueron capturados del nido para su montaje e identificación siguiendo las claves de Valverde-Monge (2010) y la descripción de Richards (1978). Dos ejemplares se depositaron en la colección entomológica del Instituto Tecnológico de Conkal, México.

En los días sin lluvia la mayor cantidad de despegues promedio fue a las 11 a.m. y la de aterrizajes alcanzó sus máximos valores entre las $13: 30$ y las 14:45, presentando su pico a las 14:00 horas (figura 1a), coincidiendo con las mayores temperaturas. En contraste, el 9 de abril tanto los despegues como los aterrizajes tuvieron su valor máximo a las 7:30 de la 
mañana, hora en la que se registró la más alta temperatura de ese día $\left(27^{\circ} \mathrm{C}\right)$, y fueron más escasos entre las 9 y las 11 horas, coincidiendo en gran medida con la lluvia (figura $1 b$ ).

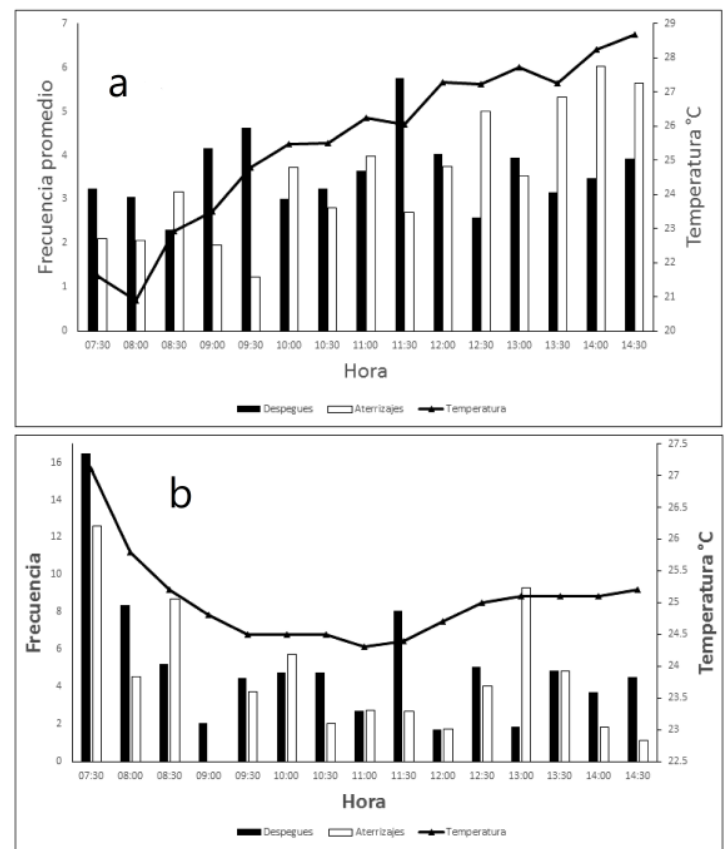

Figura 1. Actividad de forrajeo de Polybia diguetana y temperaturas en Mérida, Yucatán, México (un nido con seis días de observación). (a) Promedio de cinco días sin lluvia; (b) Día con lluvia de 9:00 a 11:45 horas.

La frecuencia de despegues presentó una débil correlación con la temperatura $(r=0,21, p=0,05)$. La frecuencia de aterrizajes se correlacionó positivamente con la temperatura $(r=0.44, p<0,00001)$ en tanto que lo hizo negativamente con la humedad relativa $(r=-0,27$, $p=0,014)$.

En los días sin lluvia se alcanzó el promedio máximo de temperaturas y mínimo de humedad relativa a las 14:00 horas con valores de $28,7^{\circ} \mathrm{C}$ y $53,8 \%$ respectivamente. A las 8:00 h se registró el mínimo de temperaturas y máximo de humedad relativa con valores de $20,9^{\circ} \mathrm{C}$ y $84,8 \%$.

El patrón de actividades del 9 de abril fue diferente del de los días sin lluvia, esto se puede deber a que ese día la humedad relativa fue muy alta $(82,5 \%$ en promedio contra $64,7 \%$ de los otros días), las temperaturas disminuyeron después de las 7:30 (figura 1b), junto con el efecto directo de la precipitación. Se sabe que la humedad relativa se correlaciona negativamente con la actividad de

forrajeo de algunos polistinos, incluso en ausencia de precipitación (Rocha y Giannotti, 2007; Hernández et al., 2009; Detoni et al., 2015).

En días húmedos y nublados la evaporación es más lenta, por lo que disminuye la necesidad de usar agua para enfriar los nidos, y puede haber un menor forrajeo por este recurso; se sabe que en otros polistinos el forrajeo por líquidos representa una alta proporción de las actividades de la colonia (Hernández et al., 2009; López et al., 2013). La precipitación tiene además un efecto directo sobre la actividad de vuelo de los insectos; en colonias del género Vespula y en otros himenópteros sociales, se ha documentado que durante las horas de lluvia la actividad de las forrajeras disminuye drásticamente, y esto se atribuye al riesgo de ser impactadas por las gotas que caen (Kasper et al., 2008).

La temperatura se correlacionó de manera directa con los despegues y con los aterrizajes, tal como sucede con otros polistinos (Elisei et al., 2005; Hernández et al., 2009; López et al., 2013; Detoni et al., 2015;). Esto se puede explicar en parte porque la temperatura se correlaciona con su tasa metabólica, por lo que a altas temperaturas se requiere de mayores cantidades de alimentos para satisfacer a la colonia (Heinrich, 1974; 1984). Por otro lado, a temperaturas muy bajas, los insectos no pueden activar sus músculos de vuelo y salir a forrajear (Heinrich, 1993; Kovac et al., 2009).

Se ha argumentado además que muchas de las presas de algunos polistinos están más activas a las horas más cálidas de día, por lo que al forrajear en esos momentos aumenta la eficiencia de captura (López et al., 2013). De igual forma, en las horas más cálidas se requiere de forrajear más agua para poder refrescar el nido; el forrajeo de este recurso puede representar más del 50 $\%$ de los viajes de algunas especies de polistinos (Hernández et al., 2009; López et al., 2013).

La temperatura tuvo un mayor efecto en las actividades de forrajeo de Polybia diguetana, que la humedad relativa; las lluvias modificaron los patrones de actividad posiblemente de manera directa por el riesgo de impacto de las gotas e indirecta, por el aumento de la humedad relativa y disminución de la temperatura.

\section{Agradecimientos}

Los autores agradecen a los revisores y editores de la revista por las sugerencias que sirvieron para mejorar el primer manuscrito. A Jonathan Hoskins por sus comentarios y al Dr. Santiago Bordera Sanjuan de la Universidad de Alicante, España por su ayuda en el montaje e identificación de los ejemplares. 


\section{Referencias}

Afik, O., Delaplane, K.S., Shafir, S., Moo-Valle, H. y Quezada-Euán, J.J.G. 2014. Nectar minerals as regulators of flower visitation in stingless bees and nectar hoarding wasps. Journal of Chemical Ecology 40: 476-483. Doi: https://doi.org/10.1007/s10886-0140455-8.

Canevazzi, N.C. de S., Noll, F.B. 2011. Environmental factors influencing foraging activity in the social wasp Polybia paulista (Hymenoptera: Vespidae: Epiponin). Psyche: A Journal of Entomology (542487) 1-8. Doi: https://doi.org/10.1155/2011/542487.

Comisión Nacional del Agua. 2010. Información Climatológica por estado. URL https://smn.cna.gob.mx/es/informacion-climatologicapor-estado. Consultado el 10 de junio de 2019.

Detoni, M., Mattos, M.D.C., Castro, M.M.D., Barbosa, B.C. y Prezoto, F. 2015. Activity schedule and foraging in Protopolybia sedula (Hymenoptera, Vespidae). Revista Colombiana de Entomología 41: 245-28.

Elisei, T., Ribeiro-Júnior, C., Guimarães, D.L. y F. Prezoto. 2005. Foraging activity and nesting of swarm-founding wasp Synoeca cyanea (Fabricius, 1775) (Hymenoptera, Vespidae, Epiponini). Sociobiology 46: 317-327.

Heinrich, B. 1993. The Hot-Blooded Insects, 1st ed. Springer Berlin Heidelberg, Heidelberg.

Heinrich, B. 1984. Strategies of thermoregulation and foraging in two vespid wasps, Dolichovespula maculata and Vespula vulgaris. Journal of Comparative Physiology B 154: 175-180. Doi: https://doi.org/10.1007/BF00684142.

Heinrich, B. 1974. Thermoregulation in endothermic insects. Science 185: 747-756.

Hernández, J., Sarmiento, C. y Fernandez, C. 2009. Actividad de forrajeo de Polybia occidentalis venezuelana (Hymenoptera, Vespidae). Revista colombiana de Entomología 35: 230-234.

Hunt, J.H. 2007. The Evolution of Social Wasps. Oxford University Press, Oxford.
Kasper, M.L., Reeson, A.F., Mackay, D.A. y Austin, A.D. 2008. Environmental factors influencing daily foraging activity of Vespula germanica (Hymenoptera, Vespidae) in Mediterranean Australia. Insectes Sociaux 55: 288295. Doi: https://doi.org/10.1007/s00040-008-1004-7.

Kovac, H., Stabentheiner, A. y Schmaranzer, S. 2009. Thermoregulation of water foraging wasps (Vespula vulgaris and Polistes dominulus). Journal of Insect Physiology 55: 959-966. Doi: https://doi.org/10.1016/j.jinsphys.2009.06.012.

López, Y., Hernández, J. y Caraballo, P. 2013. Actividad de forrajeo de la avispa social Polybia emaciata (Hymenoptera: Vespidae: Polistinae). Revista Colombiana de Entomología 39: 250-255.

Prezoto, F., Maciel, T., Detoni, M., Mayorquin, A. y Barbosa, B.C., 2019. Pest Control Potential of Social Wasps in Small Farms and Urban Gardens. Insects 10: 110. Doi: https://doi.org/10.3390/insects10070192.

Rocha, A.A. y Giannotti, E. 2007. Foraging activity of Protopolybia exigua (Hymenoptera, Vespidae) in different phases of the colony cycle, at an area in the region of the Médio São Francisco River, Bahia, Brazil. Sociobiology 50: 813-831.

Richards, O.W. 1978. The social wasps of the Americas excluding Vespinae. British Museum (Natural History). London.

Stabentheiner, A., Kovac, H., Hetz, S.K., Käfer, H. y Stabentheiner, G. 2012. Assessing honeybee and wasp thermoregulation and energetics-New insights by combination of flow-through respirometry with infrared thermography. Thermochimica Acta 534: 77-86. https://doi.org/10.1016/j.tca.2012.02.006.

StatPoint, Inc., 2007. Statgraphics Centurion XV (Versión 15.2). statgraphics.com.

Valverde-Monge, J. 2010. Clave taxonómica ilustrada para los géneros y las especies de avispas eusociales de Costa Rica (Hymenoptera: Vespidae, Polistinae). Tesis de Licenciatura. Universidad de Costa Rica. San José, Costa Rica.

Citar como: González-Moreno, A., Leirana-Alcocer, J.L. y Cervera-Herrera, J. 2019. Actividad diaria de forrajeo en Polybia diguetana Buysson 1905 (Hymenoptera, Vespidae, Polistinae). Intropica 14(2):200-203. Doi:

http://dx.doi.org/10.21676/23897864.3237. 